\title{
Fibreoptic bronchoscopy in the diagnosis of pulmonary disease in the immunocompromised host in northern Alberta
}

\author{
JenNifer A Crocket mD, Michelle R Chaput RRT, DALE C Lien MD
}

\begin{abstract}
JA CROCKET, MR CHAPUT, DC LIEN. Fibreoptic bronchoscopy in the diagnosis of pulmonary disease in the immunocompromised host in northern Alberta. Can J Infect Dis 1995;6(6):286-290.

OBJECTIVES: To determine the diagnostic utility of bronchoscopy in a population of immunocompromised hosts in northern Alberta.

PATIENTS AND METHODS: Results from bronchoscopy in 86 immunocompromised patients who underwent a total of 101 procedures were retrospectively reviewed.

RESULTS: The overall diagnostic yield was $57 \%$ with the highest yield in patients on immunosuppressive drug therapy $(80 \%)$ and the lowest yield in the group of bone marrow transplant patients $(27 \%)$.

CONCLUSIONS: Bronchoscopy is a valuable tool for the evaluation of pulmonary disease in the immunocompromised host. Overall diagnostic yield of $57 \%$ is comparable with that reported in the literature.
\end{abstract}

Key Words: Bronchoalveolar lavage, Bronchoscopy, Immunocompromised host, Pneumonia

Bronchoscopie au moyen de la fibre optique dans le diagnostic de la pneumopathie chez l'hôte immunodéficient dans le Nord de l'Alberta

OBJECTIFS : Déterminer l'utilité diagnostique de la bronchoscopie auprès d'une population de sujets immunodéficients, dans le Nord de l'Alberta.

PATIENTS ET MÉTHODES : Les résultats de la bronchoscopie effectuée chez 86 patients immunodéficients totalisant 101 interventions ont été passés en revue.

RÉSULTATS : Dans l'ensemble, le rendement diagnostique à été de $57 \%$, le rendement étant le plus fort chez les patients sous traitement médicamenteux immunosuppresseurs $(80 \%)$ et le plus faible, chez les patients ayant subi une transplantation de moelle osseuse (27\%).

CONCLUSIONS : La bronchoscopie est utile pour évaluer la pneumopathie chez l'hôte immunodéficient. Le rendement diagnostique global obtenu (57\%) se compare au rendement dont fait état la littérature.

Pulmonary Division, Department of Medicine, University of Alberta, Edmonton, Alberta

Correspondence and reprints: Dr Jennifer A Crocket, 2E4.39 Walter C MacKenzie Health Sciences Centre, University of Alberta, Edmonton, Alberta T6G 2B7. Telephone 403-492-6151, fax 403-492-6384

Received for publication October 5, 1994. Accepted June 16, 1995 
$\mathrm{B}$ OTH INFECTIOUS AND NONINFECTIOUS ETIOLOGIES MAY cause pulmonary disease in the immunocompromised host, and diagnosis can be difficult. Clinical and radiographic features are often nonspecific, and fibreoptic bronchoscopy is frequently used as a primary means of evaluation. Previous studies have assessed the utility of bronchoscopy in the diagnosis of pulmonary disease in the immunocompromised host with varied results depending on the techniques employed, population studied and diagnostic criteria used (1-10). The objective of this study was to determine the diagnostic utility of bronchoscopy in a population of immunocompromised hosts in northern Alberta by comparing bronchoalveolar lavage (BAL) with protected double-sheathed brushes, cytology brushes and transbronchial lung biopsy (TBB).

\section{PATIENTS AND METHODS}

Study population: The study population was selected retrospectively from a review of all patients who underwent bronchoscopy with BAL at the University of Alberta Hospitals, Edmonton, Alberta between August 1991 and August 1992. From a total of 143 patients, 86 immunocompromised patients who underwent a total of 101 bronchoscopy procedures were included. Fifty-seven patients of the 143 were excluded: 55 patients were not immunocompromised hosts, and in two immunocompromised patients incomplete specimens were obtained.

The study population comprised solid organ transplantation $(n=21)$, bone marrow transplantation $(n=9)$, human immunodeficiency virus (HIV) infection ( $n=28)$, immunosuppressive drug therapy $(n=10)$, hematological malignancy $(n=16)$ and lung cancer $(n=2)$ (Figure 1). Immunosuppressive drug therapy included chemotherapeutic agents, immunosuppressive drugs including cyclosporine, cyclophosphamide and azathioprine, or corticosteroid therapy taken for at least three months, the lowest dose being $10 \mathrm{mg}$ of prednisone on alternate days. Of the 86 patients, 68 were male and 18 female with a mean age of 45 years (range 18 to 84).

Bronchoscopy: Bronchoscopy was undertaken to evaluate an abnormal chest radiograph and/or pulmonary symptoms of dyspnea, cough or abnormal sputum production with increased volume or purulent coloration on 98 occasions, and for lung transplant surveillance on three occasions. The patients received standard premedication of intramuscular or intravenous atropine, and intramuscular or intravenous narcotics or diazepam. The oropharynx and upper airway were anesthestized with lidocaine solution before introduction of the bronchoscope. Protected sheath brushes $(1 \mathrm{~mm}$ microbiology brushes) were taken before BAL from the area of radiographic abnormality. All patients underwent BAL from the right middle lobe or lingula in the cases of diffuse lung disease. Where a localized radiographic infiltrate was present, the bronchoscope was wedged in a bronchus in that area. A total of $150 \mathrm{~mL}$ of normal saline in five aliquots of $30 \mathrm{~mL}$ was instilled with aspiration through the side channel of the bronchoscope after each aliquot. All five recovered aliquots were then

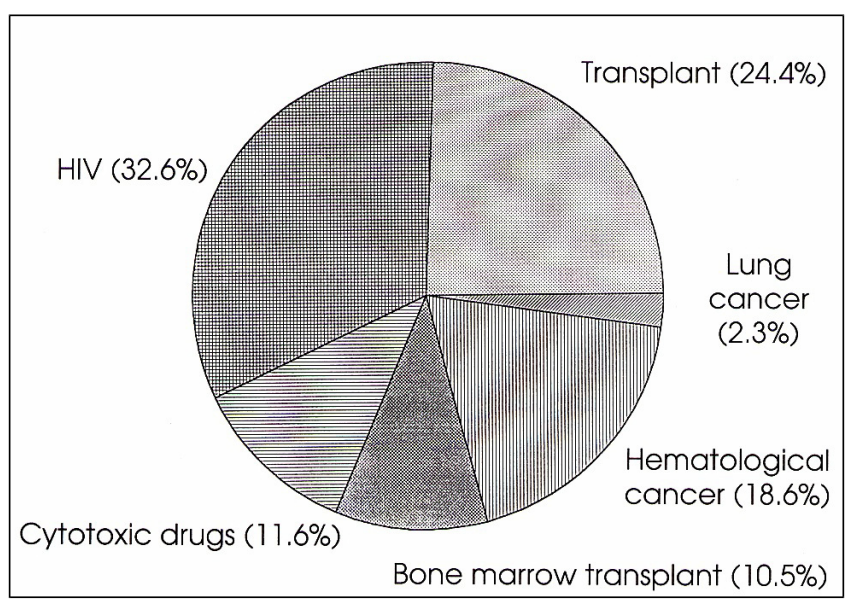

Figure 1) Distribution of the patient population ( $n=86)$. HIV Human immunodeficiency virus

combined. Following BAL, cytology brushes and TBB were obtained from the area of radiographic abnormality.

BAL specimens: BAL effluent was divided and submitted for cultures for bacteria in 99 cases, fungi in 101, mycoplasma in 95 , chlamydia in 88 , mycobacterium in 100, viruses in 87 and cytomegalovirus (CMV) in 87. CMV early antigen was detected by the shell vial technique (11-14). BAL bacterial cultures were not quantified for the numbers of bacterial colonies because this is not standard practice in the authors' laboratory. Protected double-sheathed brushes were sent for aerobic and anaerobic cultures in 89 patients and for legionella culture in 99. These brushes were transferred to Todd Hewlett and Cary Blair media with sterile technique in the bronchoscopy suite, and then vortexed and inoculated on to blood agar, chocolate agar, and anaerobic and MacConkey media in the microbiology laboratory. Sheathed brush cultures were quantified with a significant positive culture determined to be greater than $10^{6}$ colonies/L. The specimen for legionella was routinely cultured on blood agar and buffered charcoal-yeast extract media with and without antibiotics. A direct fluorescent antibody test was done if specifically requested by the physician.

Cytological examination was performed on BAL effluent in 100 cases and on brush specimens in 92. Slides were routinely stained with Papanicolaou stain, and a Gomori's methenamine-silver stain was used to identify Pneumocystis carinii and fungi. Iron stains to detect hemosiderin laden macrophages were not routinely done. TBB for histology was done in 30 patients. These tissues were processed in the hospital histology laboratory, stained with hematoxylin and eosin and interpreted by a pulmonary pathologist.

\section{RESULTS}

A positive result was obtained in $94 \%$ of procedures, but the results were determined to be clinically relevant by the authors in only $57 \%$. Clinical relevance was based on a change of therapy, institution of new therapy or confirmation of present therapy following bronchoscopy. Alternatively, results were considered clinically relevant when a positive cul- 


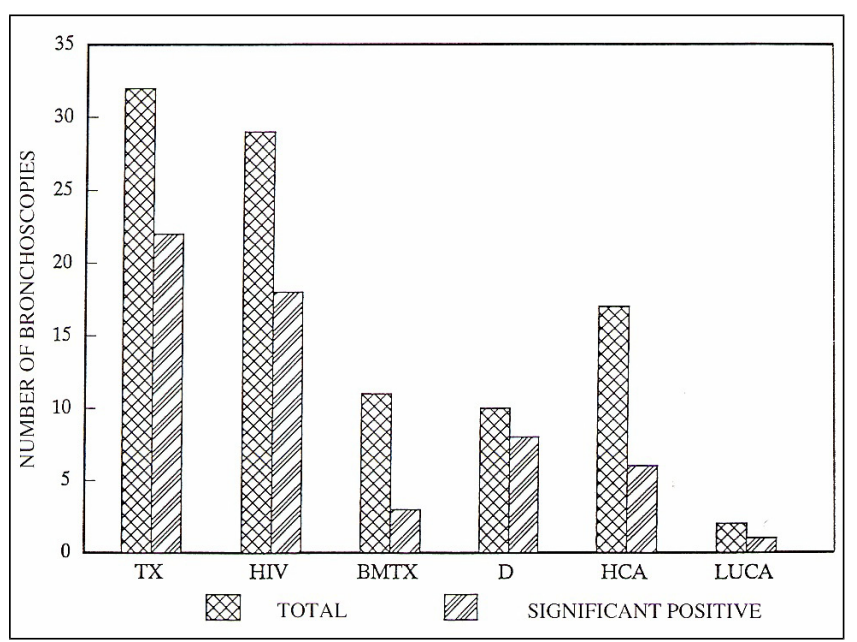

Figure 2) The number of bronchoscopy procedures that yielded results judged to be of clinical relevance. BMTX Bone marrow transplantation; D Immunosuppressive drug therapy; HCA Hematological malignancy; HIV Human immunodeficiency virus infection; LUCA Lung cancer; TX Solid organ transplantation

TABLE 1

Distribution of clinically relevant positive results*

\begin{tabular}{lrrrrrrr}
\hline & TX & BMTX & HIV & D & HCA & $\begin{array}{c}\text { LUC } \\
\text { A }\end{array}$ & Total \\
\hline Bacteria & 11 & 1 & 7 & 1 & 3 & 1 & 24 \\
Viruses & 7 & 1 & 3 & 1 & 1 & 0 & 13 \\
PCP & 0 & 0 & 9 & 2 & 0 & 0 & 11 \\
Fungi & 2 & 1 & 0 & 1 & 1 & 0 & 5 \\
Mycobacterium & 1 & 0 & 2 & 0 & 0 & 0 & 3 \\
Chlamydia & 0 & 0 & 1 & 1 & 0 & 0 & 2 \\
Mycoplasma & 0 & 0 & 3 & 0 & 0 & 0 & 3 \\
Abnormal & 3 & 0 & 0 & 2 & 1 & 0 & 6 \\
$\quad$ pathology & & & & & & & \\
Cancer & 0 & 0 & 1 & 1 & 1 & 1 & 4 \\
\hline Bonchoscopy & & & & &
\end{tabular}

*Bronchoscopy yielded more than one clinically relevant result in some patients. BMTX Bone marrow transplantation; D Immunosuppressive drug therapy; HCA Hematological malignancy; HIV Human immunodeficiency virus infection; LUCA Lung cancer; PCP Pneumocystis carinii pneumonia; TX Solid organ transplantation

ture of the same organism from other sites including blood and urine, or positive histology from biopsy or autopsy specimens was present. Positive results that were excluded were those thought to represent oropharyngeal contamination, airway colonization, CMV antigenicity without evidence of pneumonitis in the HIV group, and positive fungal cultures for Candida albicans in the absence of systemic or invasive disease. Diagnostic yield was highest in patients on immunosuppressive drug therapy $(80 \%)$ and lowest in bone marrow transplant patients $(27 \%)$. Diagnostic yield in the solid organ transplantation group was $69 \%$, in HIV patients $62 \%$, in hematological malignancy $35 \%$ and in the lung cancer group 50\% (Figure 2). Of the clinically relevant results, an infectious etiology was found in $85 \%$, noninfectious etiology in $14 \%$ and combined etiology in $1 \%$.

The diagnostic yield of BAL cultures (bacteria, fungi, mycoplasma, chlamydia, mycobacterium and viruses) was $38 \%$, of

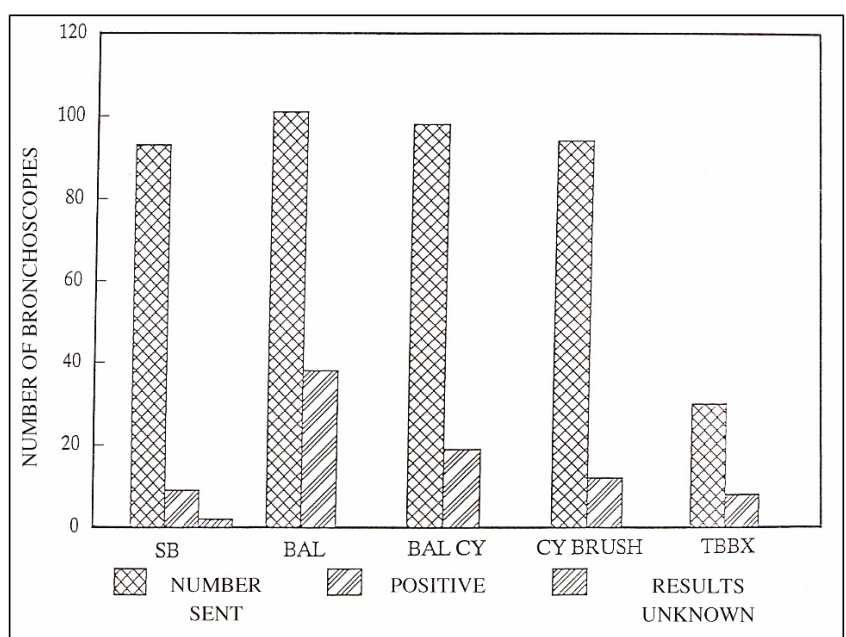

Figure 3) The diagnostic yield of sheathed brushes, bronchoalveolar lavage (BAL) cultures, BAL cytology, cytology brushes and transbronchial biopsy calculated from bronchoscopies with clinically significant results. BAL BAL cultures; BAL CY BAL cytology; CY brush Cytology brush; SB Protected sheathed brushes; TBB Transbronchial biopsy

BAL cytology was $19 \%$, of brush cytology was $13 \%$, of sheathed brush cultures (bacteria and legionella) was 10\% and of TBB was $27 \%$ (Figure 3 ). No cases of legionella were diagnosed. Positive BAL cytology results comprised malignancy in two patients, CMV infection in four patients, $P$ carinii infection in 12 patients and fungus in one patient. Cytology brushes were diagnostic for malignancy in two patients, for CMV in two patients and for $P$ carinii in eight patients. TBB was positive in eight patients: CMV in three patients, lung transplant rejection in two patients, fibrosis in one patient, a plasma cell infiltrate in one patient and talcosis in one patient.

Table 1 outlines the frequency of various diagnoses within the six patient groups. Bacteria were the most common pathogens, followed by viruses and $P$ carinii. BAL bacterial cultures were not quantified and the false positive rate was high. Of 122 positive cultures, 24 were judged to be clinically relevant. Clinically relevant bacterial isolates were Pseudomonas aeruginosa (three cases), Enterococci (three), Klebsiella pneumoniae (two), Serratia marcescens (two), Haemophilus influenzae (three), Moraxella catarrhalis (one), Streptococcus pneumoniae (six), Staphylococcus aureus (one), Neisseria meningitidis (one), coagulase-negative staphylococci (one) and mixed anaerobes (one). Of 15 positive CMV results, eight were determined to be clinically significant, and of 35 positive fungal results, five were judged to be of clinical relevance.

Differentiating fungal colonization from tissue invasion and in the case of $C$ albicans, oropharyngeal contamination, remains a difficult clinical problem. The presence of systemic or invasive disease with $C$ albicans is best confirmed by coexisting positive cultures from the other sites such as blood and urine, or by positive histology from biopsy or autopsy specimens. Of the five fungal results deemed to be clinically relevant, two represented aspergillus infection in lung transplant patients, both of whom were treated with systemic antifungal 
therapy and one of whom had autopsy evidence of invasive aspergillus. A third significant result was blastomyces in a neutropenic patient on chronic corticosteroid therapy with positive blood cultures and autopsy evidence of disseminated fungal infection. The remaining two clinically relevant results were $C$ albicans. One patient had concurrent positive blood cultures and in a second patient the result was considered relevant because the patient was treated for a prolonged time with oral antifungal therapy by the attending physician. The remaining 30 positive fungal results were $C$ albicans and thought to represent oropharyngeal contamination or airway colonization.

The complication rate of bronchoscopy was $4 \%$. Severe hypoxemia occurred in two instances (one patient recovered spontaneously while the second required mechanical ventilation). Two patients had pneumothoraces.

\section{DISCUSSION}

Pulmonary disease is a common clinical problem in the immunocompromised host and fibreoptic bronchoscopy is advocated as a primary means of evaluation allowing direct sampling from the lung with low morbidity. In this study, the overall diagnostic yield of bronchoscopy was $57 \%$. This was determined by dividing the number of positive procedures with clinically relevant results, which numbered 58 , by the total number of procedures done, of which there were 101. The diagnostic yield of BAL alone was $50 \%$, with sheathed brushes contributing additional information in three cases, brush cytology in one case and TBB in seven cases. A review of other reports in the literature indicates a diagnostic yield for BAL in similar patient groups ranging from 39 to $87 \%$ (1-10). This variability may in part be related to differences in patient populations, as suggested by Pisani and Wright (2). The yield of BAL tends to be higher in the HIV infected patient populations $(8,9)$ as opposed to patient populations with a high preponderance of hematological malignancy $(2,3)$. Our study confirms this trend with diagnostic yield being lowest in the bone marrow transplant patients $(27 \%)$ and in patients with hematological malignancy (35\%). The majority of patients in these groups had received empirical antibacterial therapy before bronchoscopy, possibly decreasing the diagnostic yield. In addition, the incidence of other diagnoses, including pulmonary hemorrhage and chemoradiation-induced lung injury, may be higher in these patient groups. Idiopathic pneumonia syndrome is another diagnostic consideration in the bone marrow transplant group (15).

In this series of patients, protected sheathed brushes added little additional information to BAL results. Bacteria were thought to represent significant pathogens in 13 cases where sheathed brush cultures were either negative or of insufficient growth to define significance. Topical lidocaine solution was used before the introduction of the bronchoscope for patient comfort. Although lidocaine is known to have antibacterial properties, there is no good information on its effect on microbiological specimens obtained by bronchoscopy. However, the potential to reduce the recovery of microorganisms in quantitative cultures must be noted.
Kahn and Jones (16) reviewed the diagnosis of bacterial respiratory infection using quantitative BAL cultures in a group of predominantly immunocompromised patients. Protected brush cultures were done concurrently in a subgroup of these patients. Their findings indicated that quantitative BAL cultures are both sensitive and specific with a reasonable correlation $(r=0.78, P<0.001)$ with protected brush cultures. They concluded that quantitative bacterial culture of the BAL specimen (in combination with analysis of BAL cell differential) can be used to diagnose bacterial respiratory tract infections. In our study sheathed brush cultures added little information to BAL bacterial cultures and the question of utility arises. It may be more cost effective to quantify BAL cultures and forego the expense of sheathed brush cultures. This issue could not be directly addressed in this study because the microbiology laboratory at our institution did not routinely quantify BAL specimens during this study period.

Legionella cultures from the protected sheath brushes were done in 99 patients with no positive cultures. Legionella is an uncommon pathogen in northern Alberta and we recommend that cultures be done only in specific patients where there is a high degree of clinical suspicion.

Brush specimens for cytological examination were done in 92 patients and yielded a clinically relevant positive result on 12 occasions. This contributed additional diagnostic information in only one patient and therefore the role of routine cytology brushing may need to be reviewed.

In this study, $57 \%$ of results obtained by bronchoscopy were clinically relevant. We defined clinical relevance as a result that caused a change in therapy, institution of new therapy or confirmation of present therapy. Defining clinical relevance was a subjective process, but perhaps best reflected the actual use of the results. Although this is a methodological limitation, it may be the best means of analyzing clinical importance. Cultures that were not clinically relevant could be considered as false positives. In assessing whether a result is of clinical relevance, probably the best guideline to offer from the review of our data is that individual results must be interpreted in light of the clinical situation. Cases in which a result is not of clinical relevance are most likely to occur where there is a high probability that the organism represents contamination from the oropharyngeal airway, airway colonization or, in the case of previous CMV exposure, persistence of the organism without active infection. In addition to clinical correlation, quantification of BAL cultures could potentially assist the clinician in distinguishing false positives from clinically relevant results.

This study has obvious limitations in that it is a retrospective analysis of a heterogeneous population of immunocompromised patients. The false negative rate for bronchoscopy could not be determined because a definitive diagnosis was not available in all patients reviewed. The study does, however, provide useful information about the diagnostic yield of bronchoscopy and the utility of certain procedures including BAL, protected double-sheathed brushes and routine cytology brushes.

We conclude that bronchoscopy is a valuable tool in our 
centre for the evaluation of pulmonary disease in the immunocompromised host. The complication rate is low compared with open lung biopsy (17), and our overall diagnostic yield of $57 \%$ is similar to other reports in the literature (1-10).

\section{REFERENCES}

1. Wallace RH, Kolbe J. Fibreoptic bronchoscopy and bronchoalveolar lavage in the investigation of the immunocompromised lung. NZ Med J 1992;105:215-7.

2. Pisani RJ, Wright AJ. Clinical utility of bronchoalveolar lavage in immunocompromised hosts. Mayo Clin Proc 1992;67:221-7.

3. Xaubet A, Torres A, Marco F, Puig-De la Bellacasa J, Faus R, Agush-Vidal A. Pulmonary infiltrates in immunocompromised patients. Chest 1989;95:130-5.

4. Williams D, Yungbluth M, Adams G, Glassroth J. The role of fiberoptic bronchoscopy in the evaluation of immunocompromised hosts with diffuse pulmonary infiltrates. Am Rev Respir Dis 1985;131:880-5.

5. Abramson MJ, Stone CA, Holmes PW, Tai EH. The role of cytomegalovirus infection in immunocompromised patients by detection of early antigen fluorescent foci. Lancet 1984;ii:1242-5.

12. Gleanes CA, Smith TF, Shuster EA, Pearson GR. Comparison of standard tube and shell vial cell culture techniques for the detection of cytomegalovirus in clinical specimens. J Clin Microbiol 1985;21:217-21.

13. Goldstein LC, McDougall J, Hackman R, Meyers JD, Thomas ED, Nowinski RC. Monoclonal antibodies to cytomegalovirus: rapid identification of clinical isolates and preliminary use in diagnosis of cytomegalovirus pneumonia. Infect Immun 1982;38:273-81. bronchoalveolar lavage in the diagnosis of suspected opportunistic pneumonia. Aust NZ J Med 1987;17:407-12.

6. Stover DE, Zaman MB, Hajdu SI, Lange M, Gold J, Armstrong D. Bronchoalveolar lavage in the diagnosis of diffuse pulmonary infiltrates in the immunocompromised host. Ann Intern Med 1984;101:1-7.

7. Heurlin N, Brattstrom C, Lonnqvist B, Wetsman L, Lidman C, Andersson J. Aetiology of pulmonary diseases in immunocompromised patients. Eur Respir J 1991;4:10-8.

8. Broaddus C, Dake MD, Stulberg MS, et al. Bronchoalveolar lavage and transbronchial biopsy for the diagnosis of pulmonary infections in the acquired immunodeficiency syndrome. Ann Intern Med 1985;102:747-52.

9. Weldon-Linne CM, Rhone DP, Bourassa R. Bronchoscopy specimens in adults with AIDS. Chest 1990;98:24-8.

10. Sobonya RE, Barbee RA, Wiens J, Trego D. Detection of fungi and other pathogens in immunocompromised patients by bronchoalveolar lavage in an area endemic for coccidioidomycosis. Chest 1990;97:1349-55.

11. Griffiths PD, Panjwani DD, Stirk PR, et al. Rapid diagnosis of

14. Stirk PR, Griffiths PD. Use of monoclonal antibodies for the diagnosis of cytomegalovirus infection by the detection of early antigen fluorescent foci (DEAFF) in cell culture. J Med Virol 1987;21:329-37.

15. Clark JG, Hansen JA, Hertz MI, et al. Idiopathic pneumonia syndrome after bone marrow transplantation. Am Rev Respir Dis 1993;147:1601-6.

16. Kahn FW, Jones JM. Diagnosing bacterial respiratory infection by bronchoalveolar lavage. J Infect Dis 1987;155:862-9.

17. Cheson BD, Samlowski WE, Tang TT, Spruance SL. Value of open-lung biopsy in 87 immunocompromised patients with pulmonary infiltrates. Cancer 1985;55:453-9. 


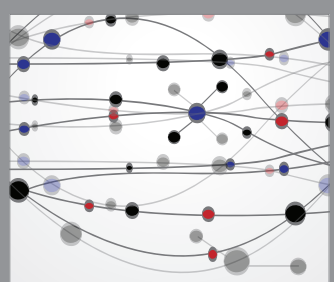

The Scientific World Journal
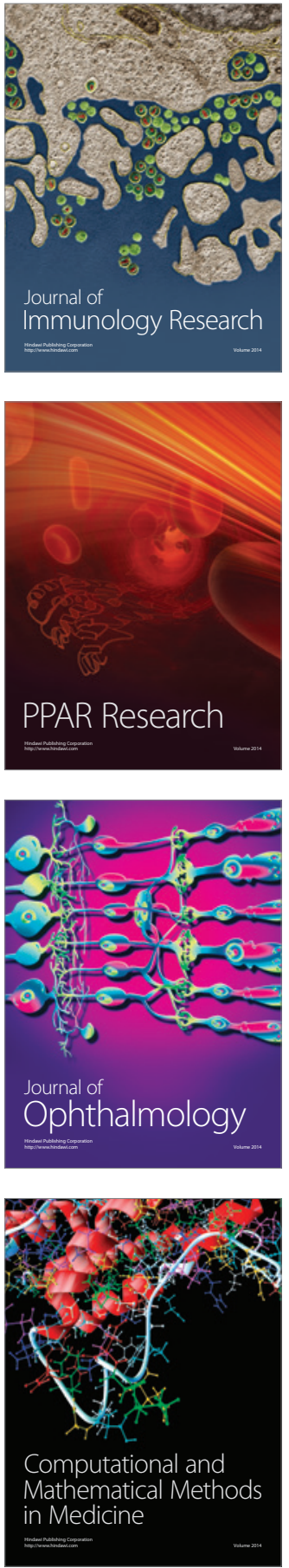

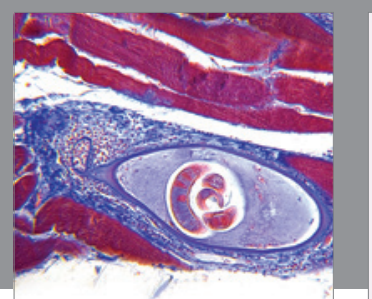

Gastroenterology Research and Practice

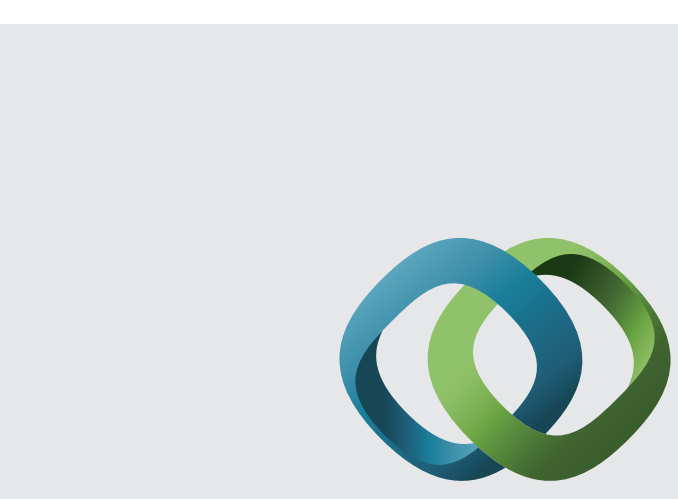

\section{Hindawi}

Submit your manuscripts at

http://www.hindawi.com
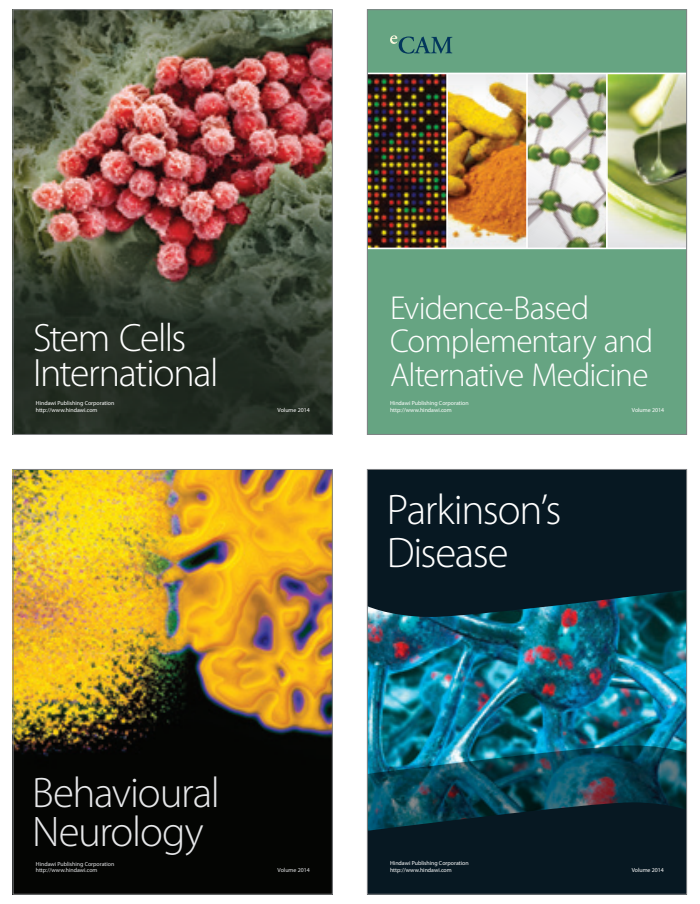
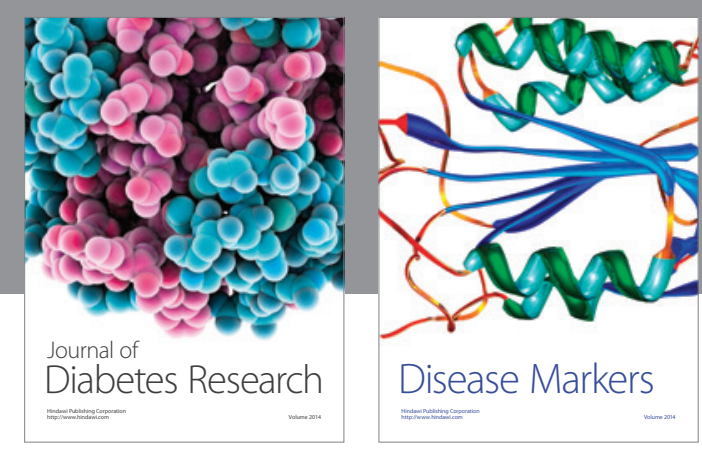

Disease Markers
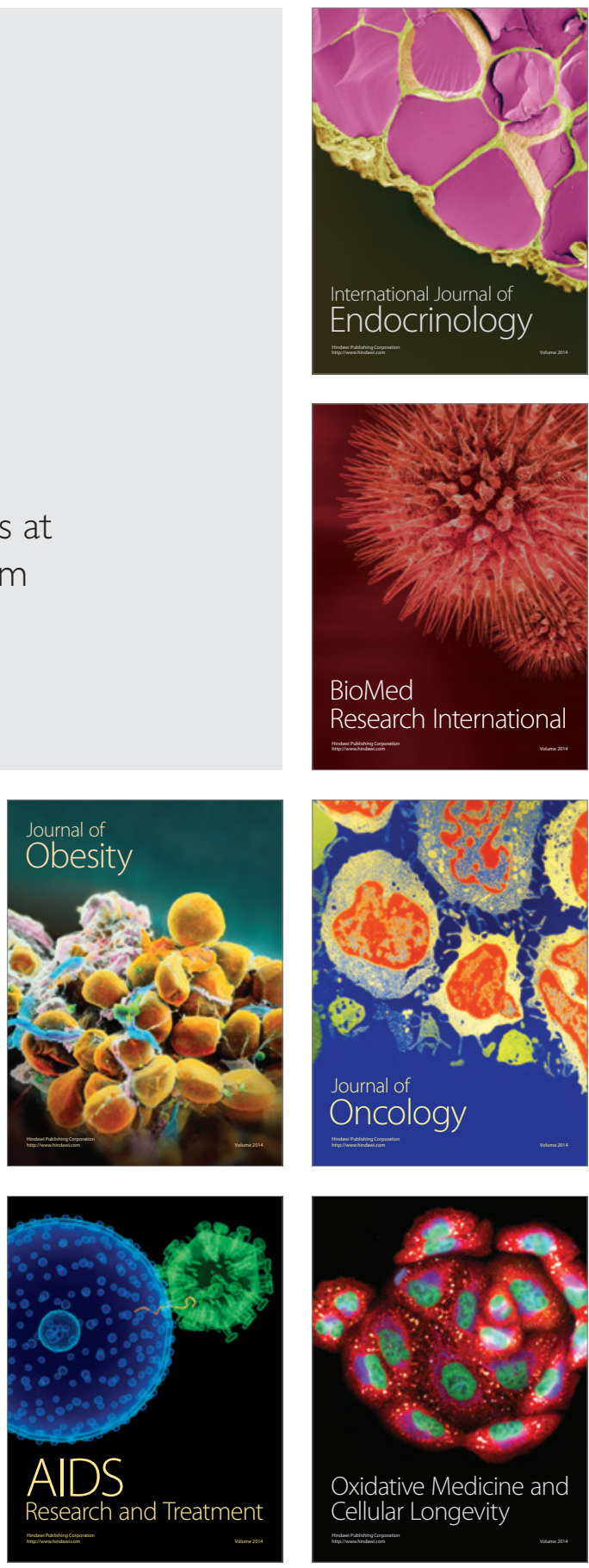